\title{
On Totally Characteristic Type Non-linear Partial Differential Equations in the Complex Domain
}

By

\author{
Hua CHEN* and Hidetoshi TAHARA**
}

\begin{abstract}
The paper deals with a singular non-linear partial differential equation $t \partial u / \partial t=F(t, x, u, \partial u / \partial x)$ with two independent variables $(t, x) \in C^{2}$ under the assumption that $F(t, x, u, v)$ is holomorphic and $F(0, x, 0,0) \equiv 0$. Set $\gamma(x)=(\partial F / \partial v)(0, x, 0,0)$. In case $\gamma(x) \equiv 0$ the equation was investigated quite well by Gérard-Tahara [3]. In case $\gamma(0)=0$ and $\operatorname{Re} \gamma^{\prime}(0)<0$ the existence of holomorphic solution was proved in Chen-Tahara [2] under a non-resonance condition. The present paper proves the existence of holomorphic solution under the same non-resonance condition but using the following weaker condition: $\gamma(0)=0$ and $\gamma^{\prime}(0) \in \boldsymbol{C} \backslash[0, \infty)$. The result is extended to higher order equations.
\end{abstract}

\section{$\S 1$. Introduction and Main Result}

Let $(t, x) \in \mathbb{C}_{t} \times C_{\backslash}$, and let us consider the following non-linear singular partial differential equation:

$$
t \frac{\partial u}{\partial t}=F\left(t, x, u, \frac{\partial u}{\partial x}\right)
$$

with $u=u(t, x)$ as an unknown function, where $F(t, x, u, v)$ is a function with respect to the variables $(t, x, u, v)$ defined in an open polydisk $\Delta$ centered at the origin of $C_{t} \times C_{x} \times C_{u} \times C_{v}$. Denote: $\Delta_{0}=\Delta \cap\{t=0, u=0, v=0\}$. We assume the following conditions:

(H-1) $F(t, x, u, v)$ is holomorphic on $\Delta$;

(H-2) $F(0, x, 0,0) \equiv 0$ on $\Delta_{0}$.

Thus the function $F(t, x, u, v)$ may be expressed in the form

$$
F(t, x, u, v)=\alpha(x) t+\beta(x) u+\gamma(x) v+\sum_{p+q+\alpha \geq 2} a_{p, q, \alpha}(x) t^{p} u^{q} v^{\alpha}
$$

Communicated by T. Kawai, January 18, 1999. Revised March 25, 1999.

1991 Math. Subject Classification(s): Primary 35A07; Secondary 35A10, 35A20.

* School of Mathematics, Wuhan University, Wuhan, 430072, China.

** Department of Mathematics, Sophia University, Tokyo, 102-8554, Japan. 
and the coefficients $\alpha(x), \beta(x), \gamma(x), a_{p, q, \alpha}(x)$ are all holomorphic functions on $\Delta_{0}$.

In general, in case $\gamma(x) \equiv 0$ the equation $\left(\mathrm{E}_{1}\right)$ is called non-linear Fuchsian type (or Briot-Bouquet type); and in case $\gamma(x) \not \equiv 0$ the equation $\left(\mathrm{E}_{1}\right)$ is called non-linear totally characteristic type. These names come from the following facts: if $\gamma(x) \equiv 0$ the linearized equation of $\left(\mathrm{E}_{1}\right)$ is linear Fuchsian type (in Baouendi-Goulaouic [1], Tahara [8]); on the other hand, if $\gamma(x) \not \equiv 0$ the linearized equation of $\left(E_{1}\right)$ is not linear Fuchsian type but is linear totally characteristic type (in the sense of Hörmander [7, section 18.3]).

Note that in case $\gamma(x) \not \equiv 0$ we have $\gamma(x)=x^{p} c(x)$ with $c(0) \neq 0$ for some $p \in \mathbb{Z}_{+}$. Denote: $\mathbb{Z}_{+}=\{0,1,2, \ldots\}$ and $\mathbb{N}=\{1,2, \ldots\}$.

The main theme is:

Problem. Under ( $\mathrm{H}-1)$ and (H-2), find a holomorphic solution $u(t, x)$ in a neighborhood of $(0,0) \in C_{t} \times C_{x}$ satisfying $u(0, x) \equiv 0$ near $x=0$.

We already know the following results.

(1) (Gérard-Tahara [3]). When $\gamma(x) \equiv 0$, if $\beta(0) \notin \mathbb{N}$, the equation $\left(\mathbb{E}_{1}\right)$ has a unique holomorphic solution $u(t, x)$ in a neighborhood of $(0,0) \in \mathbb{C}_{t} \times \mathbb{C}_{\text {、 }}$ satisfying $u(0, x) \equiv 0$.

(2) (by Cauchy-Kowalewski theorem). When $\gamma(0) \neq 0$, for any holomorphic function $\phi(t)$ with $\phi(0)=0$ the equation $\left(E_{1}\right)$ has a unique holomorphic solution $u(t, x)$ in a neighborhood of $(0,0) \in C_{t} \times \mathbb{C}_{x}$ satisfying $u(0, x) \equiv 0$ and $u(t, 0) \equiv \phi(t)$.

(3) (Chen-Tahara [2]). When $\gamma(x)=x c(x)$ with $c(0) \neq 0$, if

i) $i-\beta(0)-j c(0) \neq 0$ for any $(i, j) \in \mathbb{N} \times \mathbb{Z}_{+}$,

ii) $\operatorname{Re} c(0)<0$

hold, the equation $\left(\mathrm{E}_{1}\right)$ has a unique holomorphic solution $u(t, x)$ in a neighborhood of $(0,0) \in \mathbb{C}_{t} \times \mathbb{C}_{x}$ satisfying $u(0, x) \equiv 0$.

Remark 1. Yamane [9] has also discussed some problem concerning holomorphic solutions of $\left(\mathrm{E}_{1}\right)$ under the condition: $\gamma(x) \equiv 0$ and $\beta(0) \in \mathbb{N}$.

In this paper, by using an argument quite different from that in [2] we shall improve the above result (3) into the following form.

Theorem 1. Assume (H-1), (H-2) and that $\gamma(x)=x c(x)$ with $c(0) \neq 0$. Then, if

$$
|i-\beta(0)-j c(0)| \geq \sigma(j+1) \quad \text { for any }(i, j) \in \mathbb{N} \times \mathbb{Z}_{+}
$$

holds for some $\sigma>0$, the equation $\left(\mathrm{E}_{1}\right)$ has a unique holomorphic solution $u(t, x)$ in a neighborhood of $(0,0) \in C_{t} \times C_{x}$ satisfying $u(0, x) \equiv 0$ near $x=0$.

Theorem 1 has the following obvious corollary: 
Corollary 1. Assume (H-1), (H-2) and that $\gamma(x)=x c(x)$ with $c(0) \neq 0$. Then, if

i) $i-\beta(0)-j c(0) \neq 0$ for any $(i, j) \in \boldsymbol{N} \times \boldsymbol{Z}_{+}$,

ii) $c(0) \in C \backslash[0, \infty)$

hold, the equation $\left(\mathrm{E}_{1}\right)$ has a unique holomorphic solution $u(t, x)$ in a neighborhood of $(0,0) \in C_{t} \times C_{x}$ satisfying $u(0, x) \equiv 0$ near $x=0$.

Note that i) is a kind of non-resonance condition and ii) is the Poincaré condition on the vector field $t \partial / \partial t-c(0) x \partial / \partial x$.

We shall prove Theorem 1 in the next section, and in sections 3 and 4 we shall extend Theorem 1 to higher order totally characteristic type non-linear partial differential equations.

In sections 2 and 3 we shall use the following notations. We denote by $C[[t, x]]$ (resp. $C[[x]]$ ) the ring of formal power series in the variables $(t, x)$ (resp. in the variable $x$ ). For formal power series

$$
f(t, x)=\sum_{i, j \geq 0} f_{i, j} t^{i} x^{J}, \quad g(t, x)=\sum_{i, j \geq 0} g_{i, j} t^{i} x^{j}
$$

in $\boldsymbol{C}[[t, x]]$, we write $f(t, x) \ll g(t, x)$ if $\left|f_{i, j}\right| \leq g_{l, j}$ holds for all $(i, j) \in \boldsymbol{Z}_{+} \times \boldsymbol{Z}_{+}$, and we say that $g(t, x)$ is a majorant series of $f(t, x)$. Also we write

$$
\begin{aligned}
|f|(t, x) & =\sum_{l, J \geq 0}\left|f_{l, j}\right| t^{l} x^{j}, \\
S(f)(t, x) & =\sum_{i, j \geq 0} f_{i, J+1} t^{i} x^{J} .
\end{aligned}
$$

Clearly we have $f(t, x) \ll|f|(t, x)$,

$$
S(f)(t, x)=\frac{f(t, x)-f(t, 0)}{x}
$$

and the following: if $f(t, x)$ is convergent then $|f|(t, x)$ and $S(f)(t, x)$ are also convergent.

\section{§2. Proof of Main Result}

Under the condition $\gamma(x)=x c(x)$ with $c(0) \neq 0$ the equation $\left(\mathrm{E}_{1}\right)$ is written as

$$
t \frac{\partial u}{\partial t}=\alpha(x) t+\beta(x) u+c(x)\left(x \frac{\partial u}{\partial x}\right)+H_{2}\left(t, x, u, \frac{\partial u}{\partial x}\right)+R_{3}\left(t, x, u, \frac{\partial u}{\partial x}\right)
$$

where 


$$
\begin{aligned}
& H_{2}(t, x, u, v)=\sum_{p+q+\alpha=2} a_{p, q, \alpha}(x) t^{p} u^{q} v^{\alpha} \\
& R_{3}(t, x, u, v)=\sum_{p+q+\alpha \geq 3} a_{p, q, \alpha}(x) t^{p} u^{q} v^{\alpha} .
\end{aligned}
$$

First, let us find a formal power series solution of the form

$$
u(t, x)=\sum_{i \geq 1} u_{i}(x) t^{i}, \quad u_{i}(x) \in \mathbb{C}[[x]] \quad(\text { for } i \geq 1) .
$$

Substituting this into (2.1) and comparing the coefficients of $t^{l}$ (for $i \geq 1$ ) in the both sides of the equation, we have the following recursis or wa:

$$
\left(i-\beta(x)-c(x) x \frac{\partial}{\partial x}\right) u_{l}=f_{i-1}\left(x, u_{1}, \ldots, u_{l-1}, \frac{\partial u_{1}}{\partial x} \ldots \frac{\partial u_{l-1}}{\partial x}\right)
$$

for $i=1,2, \ldots$,

where $f_{0}(x)=\alpha(x)$ and $f_{i-1}$ (for $i \geq 2$ ) is a polynomial of $u_{1}, \ldots, u_{1-1}$ and $\partial u_{1} / \partial x, \ldots, \partial u_{i-1} / \partial x$. In particular, $f_{1}\left(x, u_{1}, \partial u_{1} / \partial x\right)$ is given by

$$
f_{1}\left(x, u_{1}, \frac{\partial u_{1}}{\partial x}\right)=H_{2}\left(1, x, u_{1}, \frac{\partial u_{1}}{\partial x}\right)
$$

Note that $(2.3)_{i}$ is expressed in the form

$$
\left(i-\beta(0)-c(0) x \frac{\partial}{\partial x}\right) u_{i}=x S(\beta)(x) u_{l}+x S(c)(x)\left(x \frac{\partial u_{l}}{\partial x}\right)-f_{l-1} .
$$

If $f_{l-1} \in \mathbb{C}\left[\left[{ }^{[} x\right]\right]$ is known and if $i-\beta(0)-c(0) j \neq 0$ for all $j \in \mathbb{Z}_{+}$, by a simple calculation we see that $(2.5)_{i}$ has a unique formal solution $u_{l}(x) \in \mathbb{C}[[x]]$.

Thus, under the condition (1.2) we can solve (2.3) inductively on $i$ and obtain a unique formal solution $u(t, x)$ of the form (2.2).

Next, let us prove the convergence of this formal solution.

Consider the following equation with respect to $Y=Y(t, x)$ :

$$
\sigma Y=A(x) t+x B(x) Y+\left|H_{2}\right|(t, x, Y, S(Y))+\left|R_{3}\right|(t, x, Y, S(Y))
$$

where $\sigma>0$ is the constant in (1.2) and

$$
A(x)=|\alpha|(x), \quad B(x)=\left|S(\beta)_{\mid}(x)+\right| S(c) \mid(x) .
$$

It is easy to see that (2.6) has a unique formal power series solution

$$
Y(t, x)=\sum_{i \geq 1} Y_{i}(x) t^{i}, \quad Y_{i}(x) \in \mathbb{C}\left[\left[x_{]}\right] \quad(\text { for } i \geq 1)\right.
$$

and the coefficients $Y_{i}(x)(i \geq 1)$ are determined by the following recursive 
formula:

(2.8) $\sigma Y_{i}=x B(x) Y_{i}+\left|f_{i-1}\right|\left(x, Y_{1}, \ldots, Y_{i-1}, S\left(Y_{1}\right), \ldots, S\left(Y_{i-1}\right)\right)$ for $i=1,2, \ldots$

which yields

$$
Y_{i}=\frac{\left|f_{i-1}\right|\left(x, Y_{1}, \ldots, Y_{i-1}, S\left(Y_{1}\right), \ldots, S\left(Y_{i-1}\right)\right)}{\sigma-x B(x)} \quad \text { for } i=1,2, \ldots
$$

Moreover we have:

Lemma 1. Under the condition (1.2) we have for all $i=1,2, \ldots$

$$
\begin{aligned}
u_{i}(x) & \ll Y_{i}(x), \\
\frac{\partial u_{i}}{\partial x}(x) & \ll S\left(Y_{i}\right)(x) .
\end{aligned}
$$

Proof. Let us prove this by induction on $i$. Put

$$
u_{i}(x)=\sum_{j \geq 0} u_{i, j} x^{J}
$$

First let us check the case $i=1$. By (1.2) and $(2.5)_{1}$ we have

$$
\begin{aligned}
& \sigma \sum_{j \geq 0}(j+1)\left|u_{1, j}\right| x^{j} \\
& \quad \ll \sum_{j \geq 0}\left|(1-\beta(0)-c(0) j) u_{1, j}\right| x^{J}=\left|\left(1-\beta(0)-c(0) x \frac{\partial}{\partial x}\right) u_{1}\right|(x) \\
& \quad \ll x|S(\beta)|(x)\left|u_{1}\right|+x|S(c)|(x)\left|x \frac{\partial u_{1}}{\partial x}\right|+\left|f_{0}\right|(x) \\
& \quad \ll x B(x) \sum_{j \geq 0}(j+1)\left|u_{1, j}\right| x^{j}+\left|f_{0}\right|(x)
\end{aligned}
$$

and therefore by (2.9) we have

$$
\sum_{j \geq 0}(j+1)\left|u_{1, j}\right| x^{j} \ll \frac{\left|f_{0}\right|(x)}{\sigma-x B(x)}=Y_{1}(x) .
$$

This easily leads us to $(2.10)_{1}$ and $(2.11)_{1}$.

Next, let $i \geq 2$ and suppose that $(2.10)_{p}$ and $(2.11)_{p}$ are already proved for all $p<i$. Then, by (1.2), (2.5) $i$ and the induction hypothesis we have 


$$
\begin{aligned}
\sigma \sum_{j \geq 0} & (j+1)\left|u_{i, j}\right| x^{j} \\
& \ll \sum_{j \geq 0}\left|(i-\beta(0)-c(0) j) u_{i, j}\right| x^{j}=\left|\left(i-\beta(0)-c(0) x \frac{\partial}{\partial x}\right) u_{i}\right|(x) \\
& \ll x|S(\beta)|(x)\left|u_{i}\right|+x|S(c)|(x)\left|x \frac{\partial u_{i}}{\partial x}\right| \\
& \quad+\left|f_{i-1}\right|\left(x,\left|u_{1}\right|, \ldots,\left|u_{i-1}\right|,\left|\frac{\partial u_{1}}{\partial x}\right|, \ldots,\left|\frac{\partial u_{i-1}}{\partial x}\right|\right) \\
& \ll x B(x) \sum_{j \geq 0}(j+1)\left|u_{1, j}\right| x^{j}+\left|f_{i-1}\right|\left(x, Y_{1}, \ldots, Y_{i-1}, S\left(Y_{1}\right), \ldots, S\left(Y_{i-1}\right)\right) .
\end{aligned}
$$

Combining this with (2.9) we obtain

$$
\sum_{j \geq 0}(j+1)\left|u_{i, j}\right| x^{j} \ll \frac{\left|f_{i-1}\right|\left(x, Y_{1}, \ldots, Y_{i-1}, S\left(Y_{1}\right), \ldots, S\left(Y_{i-1}\right)\right)}{\sigma-x B(x)}=Y_{i}(x) .
$$

This leads us to $(2.10)_{i}$ and $(2.11)_{i}$.

Lemma 1 implies that $Y(t, x)$ is a majorant series of the formal solution $u(t, x)$. Therefore, to complete the proof of Theorem 1 it is sufficient to prove that $Y(t, x)$ is convergent near $(0,0) \in C_{t} \times C_{x}$.

To do so, we write $Y(t, x)$ in the form

$$
Y(t, x)=t Y_{1}(x)+t^{2} Y_{2}(x)+t^{2} W(t, x)
$$

where

$$
W(t, x)=\sum_{i \geq 1} Y_{1+2}(x) t^{i}
$$

By the condition $\left|f_{0}\right|(x)=|\alpha|(x)=A(x)$, (2.4) and (2.9) we have

$$
\begin{aligned}
& Y_{1}(x)=\frac{A(x)}{\sigma-x B(x)} \quad(\gg 0), \\
& Y_{2}(x)=\frac{\left|H_{2}\right|\left(1, x, Y_{1}, S\left(Y_{1}\right)\right)}{\sigma-x B(x)} \quad(\gg 0) .
\end{aligned}
$$

This implies that $Y_{1}(x)$ and $Y_{2}(x)$ are holomorphic functions in a neighborhood of $x=0$ and therefore $S\left(Y_{1}\right)(x)$ and $S\left(Y_{2}\right)(x)$ are also holomorphic near $x=0$.

Let us show that $W(t, x)$ is convergent near $(0,0) \in C_{t} \times C_{x}$. By (2.4), (2.6) and (2.8) we see that $W(t, x)$ is the unique formal solution with $W(0, x) \equiv 0$ of the equation

$$
\sigma W=x B(x) W+G(t, x, t W, t S(W)),
$$


where

(2.13) $G\left(t, x, X_{1}, X_{2}\right)$

$$
\begin{aligned}
= & \left(\left|H_{2}\right|\left(1, x, Y_{1}+t Y_{2}+X_{1}, S\left(Y_{1}\right)+t S\left(Y_{2}\right)+X_{2}\right)-\left|H_{2}\right|\left(1, x, Y_{1}, S\left(Y_{1}\right)\right)\right) \\
& +\frac{1}{t^{2}}\left|R_{3}\right|\left(t, x, t Y_{1}+t^{2} Y_{2}+t X_{1}, t S\left(Y_{1}\right)+t^{2} S\left(Y_{2}\right)+t X_{2}\right) .
\end{aligned}
$$

In (2.13) we may regard $Y_{1}(x), Y_{2}(x), S\left(Y_{1}\right)(x)$, and $S\left(Y_{2}\right)(x)$ as known holomorphic functions. Since $W(t, x) \gg 0$, to have the convergence of $W(t, x)$ it is sufficient to prove

Lemma 2. If $\varepsilon>0$ is sufficiently small, the power series $W(\varepsilon \rho, \rho)$ is convergent in a neighborhood of $\rho=0 \in \boldsymbol{C}$.

Proof. Set

$$
W_{\varepsilon}(\rho)=W(\varepsilon \rho, \rho)
$$

Then, by substituting $t=\varepsilon \rho, x=\rho$ into (2.12) and by using the relation

$$
\rho S(W)(\varepsilon \rho, \rho)=W(\varepsilon \rho, \rho)-W(\varepsilon \rho, 0) \ll W(\varepsilon \rho, \rho)=W_{\varepsilon}(\rho),
$$

we have

$$
\begin{aligned}
\sigma W_{\varepsilon} & =\rho B(\rho) W_{\varepsilon}+G\left(\varepsilon \rho, \rho, \varepsilon \rho W_{\varepsilon}, \varepsilon \rho S(W)(\varepsilon \rho, \rho)\right) \\
& \ll \rho B(\rho) W_{\varepsilon}+G\left(\varepsilon \rho, \rho, \varepsilon \rho W_{\varepsilon}, \varepsilon W_{\varepsilon}\right) .
\end{aligned}
$$

Hence, instead of considering (2.14) we shall consider the following analytic equation with respect to $Z(\rho)$ :

$$
\sigma Z=\rho B(\rho) Z+G(\varepsilon \rho, \rho, \varepsilon \rho Z, \varepsilon Z) .
$$

Then, the proof of Lemma 2 can be reduced to the following lemma.

Lemma 3. If $\varepsilon>0$ is sufficiently small, the equation (2.15) has a unique formal power series solution of the form

$$
Z(\rho)=\sum_{i \geq 1} Z_{i} \rho^{l} \in \boldsymbol{C}[[\rho]]
$$

and it is convergent in a neighborhood of $\rho=0 \in C$. Moreover we have $W_{\varepsilon}(\rho) \ll$ $Z(\rho)$.

Proof. By (2.13) we see that $G\left(t, x, X_{1}, X_{2}\right)$ is an analytic function with respect to $\left(t, x, X_{1}, X_{2}\right)$ in a neighborhood of the origin of $C^{4}$ and that $G(0,0,0,0)=0$ holds. Put

$$
K=\frac{\partial G}{\partial X_{2}}(0,0,0,0)
$$


Then the equation (2.15) is written in the form

$$
(\sigma-\varepsilon K) Z=R(\rho, Z)
$$

where $R(\rho, Z)$ is a holomorphic function with respect to $(\rho, Z)$ in a neighborhood of $(0,0) \in C_{\rho} \times C_{Z}$ which depends on $\varepsilon$ and satisfies the following: $R(\rho, Z) \gg 0, R(0,0)=0$ and $(\partial R / \partial Z)(0,0)=0$.

Hence, if we choose $\varepsilon>0$ so that

$$
\sigma-\varepsilon K>0
$$

we can easily see that $(2.15)$ has a unique formal solution $Z(\rho)$ of the form (2.16). The convergence of $Z(\rho)$ is obtained by the implicit function theorem.

Moreover, by (2.14) we have

$$
(\sigma-\varepsilon K) W_{\varepsilon} \ll R\left(\rho, W_{\varepsilon}\right),
$$

and therefore by comparing (2.17) and (2.18) we obtain the result: $W_{\varepsilon}(\rho) \ll Z(\rho)$.

Thus, the proof of Lemma 3 is completed.

The proof of Theorem 1 is also completed at last.

\section{§3. Case of Higher Order Totally Characteristic $\mathrm{PDE}$}

In this section, we shall extend the result of Theorem 1 to the case of higher order totally characteristic partial differential equations.

Let $(t, x) \in C_{t} \times C_{x}, m \in N$, put $N=\#\left\{(j, \alpha) \in Z_{+} \times Z_{+} ; j+\alpha \leq m, j<m\right\}$, and denote

$$
Z=\left\{Z_{j, \alpha}\right\}_{\substack{j+\alpha \leq m \\ j<m}} \in C^{N}
$$

Let us consider

$\left(\mathrm{E}_{m}\right)$

$$
\left(t \frac{\partial}{\partial t}\right)^{m} u=F\left(t, x,\left\{\left(t \frac{\partial}{\partial t}\right)^{J}\left(\frac{\partial}{\partial x}\right)^{\alpha} u\right\}_{\substack{J+\alpha \leq m \\ J<m}}\right)
$$

with $u=u(t, x)$ as an unknown function, where $F(t, x, Z)$ is a function with respect to the variables $(t, x, Z)$ defined in an open polydisk $\Delta$ centered at the origin of $C_{t} \times C_{x} \times C_{Z}^{N}$. Denote: $\Delta_{0}=\Delta \cap\{t=0, Z=0\}$. We assume the following conditions:

$\left(\mathrm{H}_{m}-1\right) \quad F(t, x, Z)$ is holomorphic on $\Delta$;

$\left(\mathrm{H}_{m}-2\right) \quad F(0, x, 0) \equiv 0$ on $\Delta_{0}$.

Thus we rewrite $F(t, x, Z)$ near the origin as

$$
F(t, x, Z)=a(x) t+\sum_{\substack{j+\alpha \leq m \\ j<m}} b_{j, \alpha}(x) Z_{j, \alpha}+\sum_{p+|v| \geq 2} g_{p, v}(x) t^{p} Z^{v},
$$


where

$$
v=\left\{v_{J, \alpha}\right\}_{\substack{+\alpha \leq m \\ j<m}} \in Z_{+}^{N}, \quad|v|=\sum_{\substack{j+\alpha \leq m \\ j<m}} v_{j, \alpha}, \quad Z^{v}=\prod_{\substack{j+\alpha \leq m \\ j<m}}\left(Z_{J, \alpha}\right)^{v_{j} x}
$$

and the coefficients $a(x), b_{J, \alpha}(x), g_{p, v}(x)$ are all holomorphic functions on $\Delta_{0}$.

For simplicity, we write

$$
\begin{gathered}
C\left(x, t \frac{\partial}{\partial t}, \frac{\partial}{\partial x}\right)=\left(t \frac{\partial}{\partial t}\right)^{m}-\sum_{\substack{j+\alpha \leq m \\
j<m}} b_{j, \alpha}(x)\left(t \frac{\partial}{\partial t}\right)^{j}\left(\frac{\partial}{\partial x}\right)^{\alpha}, \\
D u=\left\{D_{j, \alpha} u\right\}_{\substack{j+\alpha \leq m \\
j<m}}, \quad \text { and } \quad D_{j, \alpha} u=\left(t \frac{\partial}{\partial t}\right)^{j}\left(\frac{\partial}{\partial x}\right)^{\alpha} u .
\end{gathered}
$$

Then the equation $\left(\mathrm{E}_{m}\right)$ is expressed in the form

$$
C\left(x, t \frac{\partial}{\partial t}, \frac{\partial}{\partial x}\right) u=a(x) t+\sum_{p+|v| \geq 2} g_{p, v}(x) t^{p}(D u)^{v} .
$$

If $b_{J, \alpha}(x) \equiv 0$ for all $(j, \alpha)$ with $\alpha>0, C(x, t \partial / \partial t, \partial / \partial x)$ is nothing but an ordinary differential operator in $t$ with a parameter $x$. In this case, the equation $\left(\mathrm{E}_{m}\right)$ was studied quite well in Gérard-Tahara [4]. This is the higher order version of non-linear Fuchsian type partial differential equations.

If $b_{J, \alpha}(x) \not \equiv 0$ for some $(j, \alpha)$ with $\alpha>0$, the equation $\left(\mathrm{E}_{m}\right)$ is called nonlinear totally characteristic type partial differential equations. This case is divided into the following two cases:

Case (I) $\quad b_{J, \alpha}(0) \neq 0$ for some $\alpha>0$.

Case (II) $b_{J, \alpha}(0)=0$ for all $(j, \alpha)$ with $\alpha>0$, but $b_{j, \alpha}(x) \not \equiv 0$ for some $\alpha>0$.

Gérard-Tahara [6] discussed the case (I) and proved the existence of holomorphic solutions and also singular solutions of $\left(\mathrm{E}_{m}\right)$.

Here, we shall consider a particular class of the case (II) under the following assumption:

$$
\left(\mathrm{H}_{m}-3\right) \quad b_{j, \alpha}(x)=O\left(x^{\alpha}\right) \quad(\text { as } x \rightarrow 0) \quad \text { for all }(j, \alpha) .
$$

Then, $b_{j, \alpha}(x)$ is expressed in the form $b_{j, \alpha}(x)=x^{\alpha} c_{j, \alpha}(x)$ for some holomorphic function $c_{j, \alpha}(x)$, and $C(x, t \partial / \partial t, \partial / \partial x)$ is written as

$$
\begin{aligned}
& C\left(x, t \frac{\partial}{\partial t}, \frac{\partial}{\partial x}\right) \\
& \quad=\left(t \frac{\partial}{\partial t}\right)^{m}-\sum_{\substack{j+\alpha \leq m \\
j<m}} c_{j, \alpha}(x)\left(t \frac{\partial}{\partial t}\right)^{J}\left(x \frac{\partial}{\partial x}\right)\left(x \frac{\partial}{\partial x}-1\right) \cdots\left(x \frac{\partial}{\partial x}-\alpha+1\right) .
\end{aligned}
$$


We write

$$
L(\lambda, \rho)=\lambda^{m}-\sum_{\substack{J+\alpha \leq m \\ j<m}} c_{J, \alpha}(0) \lambda^{J} \rho(\rho-1) \cdots(\rho-\alpha+1) .
$$

Theorem 2. Assume $\left(\mathrm{H}_{m}-1\right),\left(\mathrm{H}_{m}-2\right)$ and $\left(\mathrm{H}_{m}-3\right)$. Then, if

$$
|L(k, l)| \geq \sigma(k+l+1)^{m-1}(l+1) \quad \text { for any }(k, l) \in N \times \mathbb{Z}_{+}
$$

holds for some $\sigma>0$, the equation $\left(\mathrm{E}_{m}\right)$ has a unique holomorphic solution $u(t, x)$ in a neighborhood of $(0,0) \in C_{t} \times C_{x}$ satisfying $u(0, x) \equiv 0$ near $x=0$.

Denote by $c_{1}, \ldots, c_{m}$ the roots of the equation in $X$ :

$$
X^{m}-\sum_{\substack{J+\alpha=m \\ J<m}} c_{j, \alpha}(0) X^{J}=0
$$

Then, if we factorize $L(\lambda, \rho)$ into the form

$$
L(\lambda, \rho)=\left(\lambda-\xi_{1}(\rho)\right) \cdots\left(\lambda-\xi_{m}(\rho)\right),
$$

we see that

$$
\lim _{\rho \rightarrow \infty} \frac{\xi_{i}(\rho)}{\rho}=c_{l} \quad \text { for } i=1, \ldots, m
$$

Thus, as a corollary to Theorem 2 we obtain

Corollary 2. Assume $\left(\mathrm{H}_{m}-1\right),\left(\mathrm{H}_{m}-2\right)$ and $\left(\mathrm{H}_{m}-3\right)$. Then, if

i) $L(k, l) \neq 0$ for any $(k, l) \in \boldsymbol{N} \times \boldsymbol{Z}_{+}$,

ii) $c_{l} \in C \backslash[0, \infty)$ for $i=1, \ldots, m$

hold, the equation $\left(\mathrm{E}_{m}\right)$ has a unique holomorphic solution $u(t, x)$ in a neighborhood of $(0,0) \in C_{t} \times C_{x}$ satisfying $u(0, x) \equiv 0$ near $x=0$.

Remark 2. The above corollary 2 is an improvement of Theorem 2 in Chen-Tahara [2]. Recall that in [2] we have assumed the conditions $\left(\mathrm{H}_{m}-1\right)$, $\left.\left(\mathrm{H}_{m}-2\right),\left(\mathrm{H}_{m}-3\right), \mathrm{i}\right)$ and

ii) ${ }^{\prime} \operatorname{Re} c_{l}<0$ for $i=1, \ldots, m$.

Proof of Theorem 2. Denote

$$
\begin{gathered}
I=\left\{(j, \alpha) \in Z_{+} \times Z_{+} ; j+\alpha \leq m, j<m\right\}, \\
H_{2}(t, x, Z)=\sum_{p+|v|=2} g_{p, v}(x) t^{p} Z^{v}, \\
R_{3}(t, x, Z)=\sum_{p+|v| \geq 3} g_{p, v}(x) t^{p} Z^{\prime} .
\end{gathered}
$$


Assume the conditions $\left(\mathrm{H}_{m}-3\right)$ and that $b_{\jmath, \alpha}(x)=x^{\alpha} c_{J, \alpha}(x)$ for $(j, \alpha) \in I$. Then, the equation $\left(\mathrm{E}_{m}\right)$ is written in the form

$$
\begin{aligned}
& L\left(t \frac{\partial}{\partial t}, x \frac{\partial}{\partial x}\right) u \\
& \quad=x \sum_{(j, \alpha) \in I} S\left(c_{J, \alpha}\right)(x)\left(t \frac{\partial}{\partial t}\right)^{J}\left(x \frac{\partial}{\partial x}\right)\left(x \frac{\partial}{\partial x}-1\right) \cdots\left(x \frac{\partial}{\partial x}-\alpha+1\right) u \\
& \quad+a(x) t+H_{2}(t, x, D u)+R_{3}(t, x, D u) .
\end{aligned}
$$

First, let us find a formal solution of the form

$$
u(t, x)=\sum_{k \geq 1} u_{k}(x) t^{k}, \quad u_{k}(x) \in C[[x]] \quad(\text { for } k \geq 1)
$$

Substituting this into (3.3) and comparing the coefficients of $t^{k}$ (for $k \geq 1$ ) we have the following recursive formula:

$$
\begin{aligned}
& L\left(k, x \frac{\partial}{\partial x}\right) u_{k} \\
& =x \sum_{(j, \alpha) \in I} S\left(c_{J, \alpha}\right)(x) k^{J}\left(x \frac{\partial}{\partial x}\right)\left(x \frac{\partial}{\partial x}-1\right) \cdots\left(x \frac{\partial}{\partial x}-\alpha+1\right) u_{k} \\
& \quad+f_{k-1}\left(x,\left\{p^{\prime}\left(\frac{\partial}{\partial x}\right)^{\alpha} u_{p} ; 1 \leq p \leq k-1,(j, \alpha) \in I\right\}\right), \\
& \quad \text { for } k=1,2, \ldots,
\end{aligned}
$$

where $f_{0}(x)=a(x)$ and $f_{k-1}$ (for $\left.k \geq 2\right)$ is a polynomial of $\left\{p^{J}(\partial / \partial x)^{\alpha} u_{p}\right.$; $1 \leq p \leq k-1,(j, \alpha) \in I\}$.

Since $L(k, l) \neq 0$ for any $k \in N$ and any $l \in Z_{+}$, we can solve (3.5) uniquely and formally in $C[[x]]$ by induction on $k$. Thus, we have obtained a unique formal solution $u(t, x)$ of the form (3.4). It remains to prove the convergence of this formal solution.

Next, we consider the following equation with respect to $Y=Y(t, x)$ :

$$
\begin{aligned}
\sigma Y= & x C(x) Y+A(x) t \\
& +\left|H_{2}\right|\left(t, x,\left\{S^{\alpha}(Y)\right\}_{(J, \alpha) \in I}\right)+\left|R_{3}\right|\left(t, x,\left\{S^{\alpha}(Y)\right\}_{(J, \alpha) \in I}\right),
\end{aligned}
$$

where $\sigma>0$ is the constant in (3.2),

$$
A(x)=|a|(x), \quad C(x)=\sum_{(1, \alpha) \in I}\left|S\left(c_{l, \alpha}\right)\right|(x)
$$

and $S^{\alpha}(Y)$ is defined by $S^{2}(Y)=S(S(Y)), \ldots, S^{\alpha}(Y)=S\left(S^{\alpha-1}(Y)\right)$. It is easy 
to see that (3.6) has a unique formal solution $Y(t, x)$ of the form

$$
Y(t, x)=\sum_{k \geq 1} Y_{k}(x) t^{k}, \quad Y_{k}(x) \in \mathbb{C}[[x]] \quad(\text { for } k \geq 1)
$$

and the coefficients $Y_{k}(x)(k \geq 1)$ are determined by the following recursive formula:

$$
\begin{aligned}
& \sigma Y_{k}=x C(x) Y_{k}+\left|f_{k-1}\right|\left(x,\left\{S^{\alpha}\left(Y_{p}\right) ; 1 \leq p \leq k-1,(j, \alpha) \in I\right\}\right), \\
& \text { for } k=1,2, \ldots
\end{aligned}
$$

Moreover we have:

Lemma 4. Under the condition (3.2) we have for any $k=1,2, \ldots$

$$
k^{j}\left(\frac{\partial}{\partial x}\right)^{\alpha} u_{k}(x) \ll S^{\alpha}\left(Y_{k}\right)(x) \quad \text { for any }(j, \alpha) \in I .
$$

Proof. We will prove this by induction on $k$. It is easy to prove that $(3.8)_{k}$ holds for $k=1$. Let $k \geq 2$ and suppose that $(3.8)_{p}$ is already proved for all $p<k$. Denote:

$$
u_{k}(x)=\sum_{l \geq 0} u_{k, l} x^{l}, \quad Y_{k}(x)=\sum_{l \geq 0} Y_{k, l} x^{l}
$$

Also denote:

$$
U_{k}(x)=\sum_{l \geq 0}(k+l+1)^{m-1}(l+1)\left|u_{k, l}\right| x^{l} .
$$

Then, by (3.2), (3.5) and the induction hypothesis we have

$$
\begin{aligned}
\sigma U_{k}(x) & \ll\left|L\left(k, x \frac{\partial}{\partial x}\right) u_{k}\right|(x) \\
& \ll x \sum_{(j, \alpha) \in I}\left|S\left(c_{j, \alpha}\right)\right|(x) k^{j} \sum_{l \geq 0} l(l-1) \cdots(l-\alpha+1)\left|u_{k, l}\right|(x) x^{l} \\
& +\left|f_{k-1}\right|\left(x,\left\{p^{j}\left|\left(\frac{\partial}{\partial x}\right)^{\alpha} u_{p}\right|(x) ; 1 \leq p \leq k-1,(j, \alpha) \in I\right\}\right) \\
& \ll x C(x) U_{k}(x)+\left|f_{k-1}\right|\left(x,\left\{S^{\alpha}\left(Y_{p}\right) ; 1 \leq p \leq k-1,(j, \alpha) \in I\right\}\right) .
\end{aligned}
$$

Hence, combining this with (3.7) we can obtain

$$
U_{k}(x) \ll Y_{k}(x)
$$

which immediately leads us to $(3.8)_{k}$. 
By Lemma 4 we see that $Y(t, x)$ is a majorant series of the formal solution $u(t, x)$ of (3.4). Thus, to complete the proof of Theorem 2 it is sufficient to prove the convergence of $Y(t, x)$ in a neighborhood of $(0,0) \in \boldsymbol{C}_{t} \times \boldsymbol{C}_{x}$.

Now, we divide $Y(t, x)$ into

$$
Y(t, x)=t Y_{1}(x)+t^{2} Y_{2}(x)+t^{2} W(t, x),
$$

where

$$
W(t, x)=\sum_{k \geq 1} Y_{k+2}(x) t^{k}
$$

Then we have

$$
\begin{aligned}
& Y_{1}(x)=\frac{A(x)}{\sigma-x C(x)}, \\
& Y_{2}(x)=\frac{\left|H_{2}\right|\left(1, x,\left\{S^{\alpha}\left(Y_{1}\right)\right\}_{(j, \alpha) \in I}\right)}{\sigma-x C(x)},
\end{aligned}
$$

and $W(t, x)$ is the unique formal power series solution with $W(0, x) \equiv 0$ of the equation

$$
\sigma W=x C(x) W+G\left(t, x,\left\{t S^{\alpha}(W)\right\}_{(J, \alpha) \in I}\right)
$$

where

$$
\begin{aligned}
& G\left(t, x,\left\{X_{J, \alpha}\right\}_{(J, \alpha) \in I}\right) \\
&=\left\{\left|H_{2}\right|\left(1, x,\left\{S^{\alpha}\left(Y_{1}\right)+t S^{\alpha}\left(Y_{2}\right)+X_{J, \alpha}\right\}_{(J, \alpha) \in I}\right)\right. \\
&\left.-\left|H_{2}\right|\left(1, x,\left\{S^{\alpha}\left(Y_{1}\right)\right\}_{(J, \alpha) \in I}\right)\right\} \\
& \quad+\frac{1}{t^{2}}\left|R_{3}\right|\left(t, x,\left\{t S^{\alpha}\left(Y_{1}\right)+t^{2} S^{\alpha}\left(Y_{2}\right)+t X_{J, \alpha}\right\}_{(J, \alpha) \in I}\right) .
\end{aligned}
$$

Since $Y_{1}(x)$ and $Y_{2}(x)$ are holomorphic in a neighborhood of $x=0$, to prove the convergence of $Y(t, x)$ we only need to prove

Lemma 5. If $\varepsilon>0$ is sufficiently small, the power series $W\left(\varepsilon \rho^{m}, \rho\right)$ is convergent in a neighborhood of $\rho=0 \in C$.

Proof. Set

$$
W_{\varepsilon}(\rho)=W\left(\varepsilon \rho^{m}, \rho\right) .
$$

Note that the definition of $S^{\alpha}(W)$ implies

$$
S^{\alpha}(W)(t, x)=\frac{1}{x^{\alpha}}\left(W(t, x)-\sum_{j=0}^{\alpha-1} \frac{1}{j !}\left(\left(\frac{\partial}{\partial x}\right)^{J} W\right)(t, 0) x^{j}\right)
$$


and therefore we have

$$
\begin{aligned}
& \rho^{\alpha} S^{\alpha}(W)\left(\varepsilon \rho^{m}, \rho\right) \\
& \quad=W\left(\varepsilon \rho^{m}, \rho\right)-\sum_{j=0}^{\alpha-1} \frac{1}{j !}\left(\left(\frac{\partial}{\partial x}\right)^{J} W\right)\left(\varepsilon \rho^{m}, 0\right) \rho^{j} \\
& \ll W\left(\varepsilon \rho^{m}, \rho\right)=W_{\varepsilon}(\rho) .
\end{aligned}
$$

Combining this with (3.9) we obtain

$$
\sigma W_{\varepsilon} \ll \rho C(\rho) W_{\varepsilon}+G\left(\varepsilon \rho^{m}, \rho,\left\{\varepsilon \rho^{m-\alpha} W_{\varepsilon}\right\}_{(J, \alpha) \in I}\right) .
$$

Thus, if we consider the equation

$$
\sigma Z=\rho C(\rho) Z+G\left(\varepsilon \rho^{m}, \rho,\left\{\varepsilon \rho^{m-\alpha} Z\right\}_{(J . \alpha) \in I}\right),
$$

and if we choose $\varepsilon>0$ so that

$$
\sigma-\varepsilon\left(\frac{\partial G}{\partial X_{0, m}}(0,0,0)\right)>0
$$

then by the implicit function theorem we can prove that (3.10) has a unique holomorphic solution $Z(\rho)$ in a neighborhood of $\rho=0 \in C$ with $Z(0)=0$; moreover we can prove that $W_{\varepsilon}(\rho) \ll Z(\rho)$ holds. This complete the proof of Lemma 5.

Thus, the proof of Theorem 2 is completed.

\section{§4. A Generalization}

Let us consider the following two examples:

$$
\begin{gathered}
\left(t \frac{\partial}{\partial t}+x \frac{\partial}{\partial x}\right)\left(t \frac{\partial}{\partial t}+2 x \frac{\partial}{\partial x}+1\right) u=G\left(t, x, u, t \frac{\partial u}{\partial t}, \frac{\partial u}{\partial x}, t \frac{\partial^{2} u}{\partial t \partial x}, \frac{\partial^{2} u}{\partial x^{2}}\right) \\
\left(t \frac{\partial}{\partial t}\right)\left(t \frac{\partial}{\partial t}+x \frac{\partial}{\partial x}+1\right) u=G\left(t, x, u, t \frac{\partial u}{\partial t}, \frac{\partial u}{\partial x}, t \frac{\partial^{2} u}{\partial t \partial x}\right)
\end{gathered}
$$

where $G(t, x, X)$ with $X=\left(X_{0}, X_{1}, \ldots, X_{4}\right)$ or $X=\left(X_{0}, X_{1}, \ldots, X_{3}\right)$ is a holomorphic function defined in a neighborhood of $(t, x, X)=(0,0,0)$ satisfying $G(0, x, 0) \equiv 0$ and $(\partial G / \partial X)(0, x, 0) \equiv(0, \ldots, 0)$ near $x=0$.

We have:

(1) By Theorem 2 we see that (4.1) has a unique holomorphic solution $u(t, x)$ in a neighborhood of $(0,0) \in C_{t} \times \mathbb{C}_{\mathrm{r}}$ with $u(0, x) \equiv 0$.

(2) The equation (4.2) does not satisfy the condition (3.2) and so we cannot apply Theorem 2 to (4.2). Though, by a calculation we can see that 
(4.2) has also a unique holomorphic solution $u(t, x)$ in a neighborhood of $(0,0) \in C_{t} \times C_{\lambda}$ with $u(0, x) \equiv 0$.

Being motivated by this, let us give here a slight generalization of Theorem 2 so that we can apply our result to the equation (4.2).

Let $m \in N$, let $\mathscr{U}$ be a subset of $\left\{(j, \alpha) \in \boldsymbol{Z}_{+} \times \boldsymbol{Z}_{+} ; j+\alpha \leq m, j<m\right\}$, and denote

$$
Z=\left\{Z_{J, \alpha}\right\}_{(J, \alpha) \in \mathbb{I}}, \quad Z_{J, \alpha} \in C .
$$

Let us consider

$$
\left(t \frac{\partial}{\partial t}\right)^{m} u=F\left(t, x,\left\{\left(t \frac{\partial}{\partial t}\right)^{J}\left(\frac{\partial}{\partial x}\right)^{\alpha} u\right\}_{(J, \alpha) \in \|}\right) .
$$

This equation is a particular case of equations of type $\left(\mathrm{E}_{m}\right)$ and so the assumptions $\left(\mathrm{H}_{m}-1\right),\left(\mathrm{H}_{m}-2\right)$ and $\left(\mathrm{H}_{m}-3\right)$ make sense. We set

$$
\begin{gathered}
L(\lambda, \rho)=\lambda^{m}-\sum_{(J, \alpha) \in \|} c_{J, \alpha}(0) \lambda^{J} \rho(\rho-1) \cdots(\rho-\alpha+1), \\
\phi(k, l)=\max _{(,, \alpha) \in . / l}\left((k+1)^{J}(l+1)^{\alpha}\right) .
\end{gathered}
$$

Then, by the same argument as in section 3 we obtain

Theorem 3. Assume $\left(\mathrm{H}_{m}-1\right),\left(\mathrm{H}_{m}-2\right)$ and $\left(\mathrm{H}_{m}-3\right)$. Then, if

$$
|L(k, l)| \geq \sigma \phi(k, l) \quad \text { for any }(k, l) \in \boldsymbol{N} \times \boldsymbol{Z}_{+}
$$

holds for some $\sigma>0$, the equation (4.3) has a unique holomorphic solution $u(t, x)$ in a neighborhood of $(0,0) \in C_{t} \times C_{\imath}$ satisfying $u(0, x) \equiv 0$ near $x=0$.

Note that in case (4.2) we have $\mathscr{M}=\{(0,0),(1,0),(0,1),(1,1)\}$ and $L(\lambda, \rho)=\lambda(\lambda+\rho+1)$ and therefore we can apply Theorem 3 to (4.2).

\section{Acknowledgement}

The work was supported by the NNSFC and the Sophia LecturingResearch Grants of Sophia University, Tokyo.

The research was started while $\mathrm{CH}$ was a visiting professor in Department of Mathematics of Sophia University (Tokyo) during April-July 1997; he would like to thank the Institute for the invitation and its hospitality.

\section{References}

[1] Baouendi, M. S. and Goulaouic, C., Cauchy problems with characteristic initial hypersurface, Comm. Pure Appl. Math., 26 (1973), 455-475. 
[2] Chen, H. and Tahara, H., On the holomorphic solution of non-linear totally characteristic equations, Preprint 98/20, Institut für Mathematik, Potsdam, 1998.

[ 3 ] Gérard, R. and Tahara, H., Holomorphic and singular solutions of nonlinear singular first order partial differential equations, Publ. RIMS, Kyoto Univ., 26 (1990), 979-1000.

[4] — Solutions holomorphes et singulières d'équations aux dérivées partielles singulières non linéaires, Publ. RIMS, Kyoto Univ., 29 (1993), 121-151.

[5] —-, Singular nonlinear partial differential equations, Vieweg, 1996.

[6] - Holomorphic and singular solutions of non-linear singular partial differential equations, II. "Structure of differential equations, Katata/Ky'oto, 1995" (edited by MorimotoKawai), 135-150, World Scientific, 1996.

[7] Hörmander, L., The analysis of linear partial differential operators III, Springer, 1985.

[ 8 ] Tahara, H., Fuchsian type equations and Fuchsian hyperbolic equations, Japan. J. Math., 5 (1979), 245-347.

[9] Yamane, H., Nonlinear singular first order partial differential equations whose characteristic exponent takes a positive integer value, Publ. RIMS, Kyoto Univ., 33 (1997), 801-811. 